\title{
Adaptación transcultural de un cuestionario para evaluar la autopercepción de la práctica clínica basada en la evidencia. Una experiencia con licenciadas en obstetricia
}

Cross-cultural adaptation of a questionnaire to assess the self-perception of evidence-based clinical practice. An experience with midwives

Gabriela Paola Ammatuna ${ }^{a}$, Sergio Adrián Terrasa ${ }^{\mathrm{b}, \mathrm{c}, \mathrm{d}}$, Fernando Ramón Vázquez Peña ${ }^{\mathrm{b}}$

\begin{abstract}
Resumen
Antecedentes. Dado que algunas intervenciones clínicas tienen un efecto contraproducente y hasta opuesto al deseado, es necesario contar con instrumentos para evaluar la actitud de los profesionales de la salud hacia la práctica clínica basada en la evidencia (PCBE).

Objetivos. Adaptar transculturalmente al español rioplatense y validar psicométricamente un cuestionario que permita investigar la percepción de licenciadas en obstetricia hacia la PCBE. Describir la percepción de una muestra de este colectivo profesional.

Métodos. Realizamos una adaptación transcultural del cuestionario EBPQ-19, un análisis de su validez de constructo a través de análisis factorial exploratorio y de su confiabilidad, mediante el coeficiente Alpha de Cronbach, y evaluamos la percepción de las licenciadas en obstetricia hacia la PCBE a través de la administración del instrumento obtenido a 150 profesionales matriculadas en el Colegio de Obstétricas V del partido de Quilmes, Buenos Aires.

Resultados. Luego de adaptar lingüísticamente tres ítems para optimizar su desempeño en nuestra región, documentamos adecuadas propiedades psicométricas del EBPQ-19. Los ítems 1, 2, 3, 4 y 5 (dominio 'prácticas') cargaron en un factor; los ítems 6, 7 y 8 (dominio 'actitud') presentaron cargas factoriales mayores a 0,79 en un mismo factor; y los ítems 10, $11,12,13,14$ y 15 (dominio 'habilidades y competencias') presentaron cargas mayores a 0,45 en un tercer factor. Los coeficientes Alpha de Cronbach de los tres factores y el general fueron mayores a 0,8. Más del $95 \%$ de las respuestas mostraron compromiso para con las pacientes respecto de buscar información para resolver dudas clínicas, pero $94 \%$ de las encuestadas considera no tener habilidades y conocimientos para ejercer una PCBE.

Conclusiones. La versión adaptada del EBPQ-19 tiene buen desempeño y alentamos su utilización para la evaluación y el monitoreo de programas educativos que promuevan la PCBE.
\end{abstract}

\begin{abstract}
Background. Since some clinical interventions may have a counterproductive and even opposite effect, instruments to assess the attitude of health professionals towards evidence-based clinical practice (EBCP) are necessary.

Objectives. To cross-culturally adapt to Rioplatense Spanish and to validate psicometrically a questionnaire that allows to investigate the perception of Graduates in Obstetrics/Midwifery towards EBCP and to describe the perception of a sample of this healthcare professionals.

Methods. We performed a cross-cultural adaptation of the EBPQ-19 questionnaire, and analyzed its construct validity through exploratory factor analysis, and its reliability, using Cronbach's Alpha coefficient. Then we evaluated the perception of the Graduates in Obstetrics towards the EBCP through the administration of the instrument to 150 professionals enrolled in the College of Midwives V in Quilmes, Buenos Aires.

Results. After linguistically adapting three items to adapt their performance in our region, we documented satisfactory psychometric properties of EBPQ-19. Items 1, 2, 3, 4 and 5 ('practices' domain) loaded in one factor; items 6, 7 and 8 ('attitude' domain) had factorial loads greater than 0.79 in the same factor; and items 10, 11, 12, 13, 14 and 15 ('skills and competencies') had factorial loads greater than 0.45 in a third factor. General and factor-specific Cronbach's alpha coefficients were greater than 0.8 . More than $95 \%$ of the responses revealed commitment to patients regarding seeking information to resolve clinical questions, but $94 \%$ of the subjects considered they had no skills and knowledge to deliver a EBCP.

Conclusions. The adapted version of the EBPQ-19 has a good performance. We encourage its use for the evaluation and monitoring of educational programs that advocate for an EBCP.
\end{abstract}

Palabras clave: Partería, Medicina Basada en la Evidencia, Encuestas y Cuestionarios. Keywords: Midwifery, Evidence-Based Medicine, Surveys and Questionnaires.

Ammatuna G, Terrasa S, Vazquez Peña FR. Adaptación transcultural de un cuestionario para evaluar la autopercepción de la práctica clínica basada en la evidencia. Una experiencia con licenciadas en obstetricia. Evid Actual Pract Ambul. 2019;22(3):e002028.

\footnotetext{
a Colegio de Obstétricas V de la Provincia de Buenos Aires. ammatunag@yahoo.com

b Servicio de Medicina Familiar y Comunitaria, Hospital Italiano de Buenos Aires. fernandoramon.vazquez@hospitalitaliano.org.ar

c Departamento de Investigación, Hospital Italiano de Buenos Aires.

d Departamento de Salud Pública, Instituto Universitario Hospital Italiano de Buenos Aires. sergio.terrasa@hospitalitaliano.org.ar
} 


\section{Introducción}

Desde principios del siglo XX, la estrategia educativa en obstetricia ha estado sesgada hacia la intervención en la asistencia del parto, promoviendo en estudiantes de la Licenciatura en Obstetricia el uso de procedimientos basados en la persuasión y las políticas sanitarias de turno, más que en el uso de la evidencia científica. Sin embargo, hoy contamos con evidencia de que algunas prácticas tienen un efecto contraproducente y hasta opuesto al deseado. Por ejemplo, el estrés ocasionado por el uso de oxitocina $^{1,2}$, opiáceos ${ }^{3,4}$, analgesia epidural ${ }^{5-11}$ y amniotomía, se asocia a trabajos de parto más largos ${ }^{12}$ y a patrones de latido cardíaco fetal potencialmente vinculados a reducción de la perfusión y la oxigenación del bebé ${ }^{13}$. Por otro lado, los hijos de madres que habían recibido barbitúricos, opiáceos y/o gas óxido nitroso durante el parto habrían sido 4,7 veces más propensos a ser adictos en la edad adulta ${ }^{14}$; mientras que la operación cesárea involucra una cirugía abdominal mayor, incrementando entre dos y cuatro veces el riesgo de muerte materna ${ }^{15,16}$. Además, una cesárea previa aumenta el riesgo para la madre y para la salud del bebé en los embarazos siguientes, y puede ocasionar infertilidad y embarazos ectópicos, muertes fetales inexplicables y problemas placentarios, incluyendo desprendimiento de placenta $^{17,18}$.

Lo expuesto nos lleva a considerar la necesidad de repensar las estrategias de educación profesional hacia las estudiantes de la licenciatura en Obstetricia ${ }^{19,20}$, para que promuevan una práctica clínica basada en la evidencia (PCBE). Sin embargo, trasladar a la práctica clínica la mejor evidencia disponible suele ser dificultoso, ya que la PCBE no es la aplicación a ciegas de consejos recopilados a partir de la bibliografía publicada recientemente a problemas de pacientes individuales; sino que requiere el uso de una serie de pasos para recopilar información lo suficientemente útil para responder a una pregunta elaborada de manera meticulosa para un paciente determinado ${ }^{21}$.

Todo intento institucional que pretenda desarrollar un ciclo de mejora de la calidad de la atención a través de la implementación de una PCBE requiere de herramientas para la evaluación de la situación basal de los profesionales involucrados y para poder monitorear las respuestas a las intervenciones de mejora, para así planificar las correcciones necesarias. Si bien, han sido publicados varios instrumentos para evaluar la actitud de los profesionales de la salud hacia la $\mathrm{PCBE}^{22}$, se destaca el publicado en 2006 por Upton et $\mathrm{al}^{23}$, posteriormente traducido al español, adaptado transculturalmente y validado en una muestra de 324 individuos por De Pedro Gómez et al ${ }^{24}$. Esta escala es una herramienta práctica y breve que permite evaluar en forma directa la predisposición y la voluntad de los/las profesionales para proveer una PCBE.

El objetivo de este trabajo fue obtener una versión válida del EBPQ-19, adaptada a la lengua castellana de Argentina en una muestra de Licenciadas en Obstetricia. Además, nos propusimos evaluar la percepción que tiene este colectivo de trabajadoras acerca de su práctica clínica.

\section{Métodos}

\section{Diseño de investigación}

Estudio descriptivo, de corte transversal. Los datos fueron recolectados en forma prospectiva.

\section{El cuestionario de Efectividad Clínica y Práctica Basada en la Evidencia (EBPQ-19)}

El cuestionario de Efectividad Clínica y Practica Basada en la Evidencia (EBPQ-19) es auto-administrado y consta de 19 ítems de respuesta tipo Likert con siete opciones cada uno, estructurados en tres factores que se corresponden a sendas dimensiones respecto de la PCBE: práctica (6 ítems), actitud (3 ítems) y conocimientos y habilidades (10 ítems).

Cada ítem puntúa de 1 a 7 , siendo 1 el valor menos favorable y 7 , el más favorable, en términos de la competencia percibida en la aplicación de PCBE. El rango de los resultados de la escala oscila entre 19 y 133 puntos.

\section{1) Adaptación transcultural del cuestionario EBPQ-19}

Este proceso fue realizado de acuerdo a las recomendaciones metodológicas del consenso ISPOR para la Traducción y Adaptación Transcultural publicado por Wild et $\mathrm{al}^{25}$ y de la revisión de Ramada Rodilla et al $^{26}$.

\section{Adaptación de cuestionario al lenguaje del Área Metropolitana de} Buenos Aires (AMBA)

Dos investigadores llevaron a cabo este primer paso, con el lenguaje del AMBA como lengua nativa y con conocimientos de la disciplina de las personas que serían blanco del instrumento.

\section{Reconciliación de las dos primeras versiones adaptadas al} lenguaje del AMBA y comparación de esta versión resultante con el instrumento original

Ambos investigadores compararon las dos versiones entre sí con el instrumento original, evaluando discrepancias en la formulación de las preguntas, así como las equivalencias conceptuales, semánticas y de contenido del instrumento traducido. Hicieron luego una lectura final en busca de errores gramaticales o de ortografía (Proof Reading).

\section{Entrevistas cognitivas}

Se llevaron a cabo con una muestra de diez participantes, con un consentimiento informado oral. Se usó el formato de 'pensar en voz alta', y se invitó a las entrevistadas a comentar las dificultades que iban percibiendo mientras completaban el cuestionario. Todas las entrevistas fueron grabadas y analizadas.

\section{Revisión de los resultados de las entrevistas cognitivas y} formulación de la versión final

La investigadora principal analizó la información obtenida de las entrevistas cognitivas y luego generó la versión final del cuestionario.

\section{Lectura final en busca de errores gramaticales o de ortografía (Proof Reading)}

Una vez resueltos todos los ítems, la investigadora principal formuló la versión final del instrumento, describiendo las decisiones tomadas en términos de traducción y contenidos.

\section{2) Análisis de la validez de constructo y la confiabilidad}

Para el análisis de la validez de constructo del cuestionario fue empleado análisis factorial exploratorio (AFE). La confiabilidad fue evaluada a través de la consistencia interna (coeficiente alpha de Cronbach), tanto general como por factor. Basándonos en la propuesta de Streiner ${ }^{27}$ y procurando contar con por lo menos cinco observaciones por ítem del cuestionario, estimamos un tamaño muestral mínimo de 100 personas encuestadas.

\section{3) Evaluación de la percepción de las Licenciadas en Obstetricia} y estudiantes de Obstetricia hacia la PCBE

Fue realizada a través de una encuesta auto-administrada, aplicando el instrumento en español EBPQ-19, validado al lenguaje español del AMBA de acuerdo al procedimiento descripto en el paso (1). Al instrumento resultante se le agregó al principio un breve cuestionario que indagó sobre datos demográficos, laborales y académicos; y el consentimiento informado.

Para el cálculo del tamaño muestral utilizamos la fórmula propuesta por Hayes et al ${ }^{28}: \mathbf{n}=\left(\mathbf{t}^{2} \mathbf{x} \mathbf{D E}^{2}\right) / \mathbf{E T}^{2}$, en la que T es el nivel de confianza, DE es la desviación estándar y ET, el error tolerable. De acuerdo a este autor, decidimos trabajar con un nivel 
de confianza de $95 \%$ (t: 1,96) y con un error tolerable de 0,20. Asumiendo que la desviación estándar del puntaje de cada ítem sería de 1,2, estimamos que deberíamos contar con 150 participantes. Los criterios de inclusión fueron: tener entre 25 y 65 años de edad, poseer una licenciatura en obstetricia y encontrarse matriculada en el V Colegio de Obstétricas del partido de Quilmes, Provincia de Buenos Aires.

\section{Estrategias para minimizar los sesgos}

Para minimizar el sesgo de cortesía, los cuestionarios fueron entregados para ser auto-administrados por los participantes en forma anónima, asegurándoles la confidencialidad de los datos.

\section{Análisis estadístico}

Se realizó un análisis descriptivo de las variables agrupando los resultados de cada uno de los ítems del cuestionario, de acuerdo a los dominios propuestos por los autores del cuestionario original: 1) actitudes, 2) conocimiento, 3) habilidades y prácticas.

\section{Aspectos éticos}

Se obtuvo la autorización al Comité de investigación Científica y de Ética profesional del Colegio de Obstétricas, distrito $V$ del partido de Quilmes, provincia de Buenos Aires. Ninguno de los participantes de este estudio recibió compensación económica y todos completaron el cuestionario en forma individual. Se consideró que quién luego de haber leído la información proporcionada en el consentimiento informado que precedía al cuestionario comenzara a llenarlo, estaba consintiendo participar.

\section{Resultados}

\section{1) Adaptación transcultural del cuestionario original}

El instrumento fue ligeramente modificado en los tres ítems que se describen en la Tabla 1, para optimizar su interpretación en el idioma español hablado en argentina.

Tabla 1. Resultado de la adaptación transcultural de tres de los ítems del cuestionario original.

\begin{tabular}{|l|l|}
\hline \multicolumn{1}{|c|}{ Item original } & \multicolumn{1}{|c|}{$\begin{array}{c}\text { Item resultante de la } \\
\text { adaptación transcultural }\end{array}$} \\
\hline $\begin{array}{l}\text { ¿Con qué frecuencia se ha hecho } \\
\text { las siguientes cuestiones para } \\
\text { responder alguna posible laguna } \\
\text { surgida en su conocimiento? }\end{array}$ & $\begin{array}{l}\text { ¿Con que frecuencia se ha } \\
\text { hecho preguntas con el } \\
\text { objetivo de contestar dudas en } \\
\text { su conocimiento? }\end{array}$ \\
\hline $\begin{array}{l}\text { Formulé una pregunta de } \\
\text { búsqueda claramente definida, } \\
\text { como el principio del proceso para } \\
\text { cubrir esta laguna. }\end{array}$ & $\begin{array}{l}\text { Formulé una pregunta de } \\
\text { búsqueda claramente definida } \\
\text { para ayudarme a contestar una } \\
\text { duda en mis conocimientos. }\end{array}$ \\
\hline $\begin{array}{l}\text { Me aferro a métodos probados y } \\
\text { fiables más que cambiar a } \\
\text { cualquier cosa nueva. }\end{array}$ & $\begin{array}{l}\text { Prefiero métodos probados y } \\
\text { fiables más que cambiar a } \\
\text { cualquier cosa nueva. }\end{array}$ \\
\hline
\end{tabular}

\section{2) Análisis de la validez de constructo y la confiabilidad del cuestionario}

Mediante el análisis de los resultados de las encuestas realizadas a 150 licenciadas en Obstetricia que manifestaron trabajar todas en ambos ámbitos de interés (privado y estatal) y haber recibido al menos 20 horas de educación sobre PCBE, se realizó el análisis de su validez de constructo y de su confiabilidad.

\section{Validez de constructo}

Se realizó el AFE en el que se evidenció que uno de los items ('Habilidades para la investigación') presentaba cargas significati- vas en todos los factores. Analizando conceptualmente el contenido de este item, este hallazgo resultó lógico, ya que sería esperable que la persona que manifieste tener habilidades para la investigación también tendría un puntaje alto en la mayoría de los demás items del cuestionario, y a la inversa. Por este motivo se decidió eliminar esta pregunta, calificada como transversal, para poder analizar adecuadamente la estructura factorial del instrumento.

Tabla 2. Resultado del análisis factorial exploratorio a través de una matriz de componentes principales rotados (normalización Varimax con Kaiser luego de 14 iteraciones).

\begin{tabular}{|c|c|c|c|}
\hline Item & $\begin{array}{c}\text { Componente } 1 \text { - } \\
\text { prácticas }\end{array}$ & $\begin{array}{c}\text { Componente } \\
2 \text { - } \\
\text { Habilidades y } \\
\text { competencias } \\
\end{array}$ & $\begin{array}{l}\text { Componente } \\
3 \text { - Actitud }\end{array}$ \\
\hline 1 & 0,718 & & \\
\hline 2 & 0,468 & & 0,739 \\
\hline 3 & 0,474 & & 0,721 \\
\hline 4 & 0,923 & & \\
\hline 5 & 0,923 & & \\
\hline 6 & 0,538 & & 0,791 \\
\hline 7 & & & 0,928 \\
\hline 8 & & & 0,942 \\
\hline 9 & & 0,892 & \\
\hline 10 & & 0,810 & \\
\hline 11 & & 0,778 & \\
\hline 12 & 0,486 & 0,756 & \\
\hline 13 & & 0,726 & 0,418 \\
\hline 14 & 0,711 & 0,592 & \\
\hline 15 & 0,627 & 0,476 & \\
\hline
\end{tabular}

Al eliminar el item mencionado, en el AFE se conformó una estructura de tres factores. Se verificaron cargas factoriales significativamente bajas (menores de 0,4 ) en tres ítems ('Evalué críticamente mediante criterios explícitos, las referencias bibliográficas halladas', 'Conocimiento de cómo recuperar evidencia de distintas fuentes' y 'Capacidad para determinar la validez del material encontrado'). Luego de estudiar el contenido de cada una y entender que no eran especialmente relevantes para la herramienta, estas preguntas fueron eliminadas.

La estructura factorial de tres factores se mantuvo en la versión final del instrumento, con las siguientes características (ver Tabla 2 y material suplementario).

- Dominio prácticas: Los ítems 1, 2, 3, 4 y 5, correspondientes a este dominio cargaron en un factor. Los ítems 1, 4 y 5 tuvieron cargas factoriales altas (mayores a 0,7 ). Los ítems 2 y 3 presentaron cargas factoriales mayores a 0,4 sin embargo 'cabalgaron' también en el factor o dominio denominado 'Actitud' con cargas significativas. 
- Dominio actitud: Los ítems 6, 7 y 8, correspondientes a este dominio presentaron cargas factoriales muy altas (mayores a 0,79 ) en un mismo factor.

- Dominio habilidades y competencias: Los ítems 9, 10, 11, $12,13,14$ y 15, correspondientes a este dominio cargaron en un factor con cargas siempre mayores a 0.45 . Sin embargo, los ítems 14 y 15 cargaron significativamente también en el factor o dominio denominado 'Prácticas'.

\section{Confiabilidad}

Tanto los coeficientes Alpha de Cronbach de cada factor como el global, fueron óptimos. El factor 1 (equivalente al dominio 'Practicas') tuvo un resultado de 0,88 ; el factor 2 (equivalente al dominio 'Habilidades y competencias'), de 0,81 ; y el factor 3 (correspondiente al dominio 'Actitud'), un valor de 0,90. Por último, el Alpha de Cronbach general fue de 0.83 .

\section{3) Percepción del colectivo de trabajadoras acerca de su práctica clínica \\ Dominio prácticas}

Las respuestas obtenidas al interrogar sobre la frecuencia con que las licenciadas en obstetricia encuestadas habían formulado preguntas con el objetivo de contestar alguna duda en sus conocimientos al asistir a algún paciente en el último año se muestran en la Tabla 3.

Tabla 3. Respuestas en el dominio prácticas de la versión Argentina del cuestionario EBPQ-19 de las licenciadas en obstetricia matriculadas en el Colegio de Obstétricas $V$ del partido de Quilmes, Buenos Aires $(\mathrm{N}=150)$. Nota: Escala del 1 al 7 , en la que $1=$ nunca y $7=$ frecuentemente.

\begin{tabular}{|l|c|c|c|}
\hline Pregunta & $\begin{array}{c}\text { Puntaje } 7, n \\
(\%)\end{array}$ & $\begin{array}{c}\text { Puntaje 6, } \\
n(\%)\end{array}$ & $\begin{array}{c}\text { Puntaje } \\
50 \\
\text { inferior, } \\
n(\%)\end{array}$ \\
\hline $\begin{array}{l}\text { 1. Formulé una pregunta } \\
\text { de búsqueda claramente } \\
\text { definida para ayudarme } \\
\text { a contestar una duda en } \\
\text { mis conocimientos }\end{array}$ & $109(72,3)$ & $41(27,3)$ & 0 \\
$\begin{array}{l}\text { 2. Indagué la eviden- } \\
\text { cia relevante después de } \\
\text { haber elaborado la pre- } \\
\text { gunta }\end{array}$ & $98(65,3)$ & $52(34,7)$ & 0 \\
$\begin{array}{l}\text { 3. Integré la evidencia } \\
\text { encontrada con mi expe- } \\
\text { riencia }\end{array}$ & $97(64,7)$ & $53(35,3)$ & 0 \\
$\begin{array}{l}\text { 4. Evalué los resultados } \\
\text { de mi practica }\end{array}$ & $129(86,0)$ & $21(14,0)$ & 0 \\
$\begin{array}{l}\text { 5. Compartí esta infor- } \\
\text { mación con mis colegas }\end{array}$ & $129(86,0)$ & $21(14,0)$ & 0 \\
\hline
\end{tabular}

\section{Dominio actitud}

Las respuestas obtenidas al indagar en qué lugar de la escala se situarían las profesionales encuestadas para cada uno de los enunciados vinculados con el dominio 'actitud' se resumen en la Tabla 4.
Tabla 4. Respuestas en el dominio actitud de la versión Argentina del cuestionario EBPQ-19 de las licenciadas en obstetricia matriculadas en el Colegio de Obstétricas $\vee$ del partido de Quilmes, Buenos Aires $(\mathrm{N}=150)$. Nota: valor mínimo=1, valor máximo=7.

\begin{tabular}{|l|c|c|c|}
\hline Pregunta & $\begin{array}{c}\text { Puntaje 7, } \\
n(\%)\end{array}$ & $\begin{array}{c}\text { Puntaje } \\
6, n(\%)\end{array}$ & $\begin{array}{c}\text { Puntaje 5 o } \\
\text { inferior, } n \\
(\%)\end{array}$ \\
\hline $\begin{array}{l}\text { 6. Recibo de buen agra- } \\
\text { do preguntas sobre mi } \\
\text { práctica clínica }\end{array}$ & $69(46,0)$ & $60(40,0)$ & $21(14,0)$ \\
$\begin{array}{l}\text { 7. La práctica basada en } \\
\text { la evidencia es funda- } \\
\text { mental para la práctica } \\
\text { profesional }\end{array}$ & $70(46,7)$ & $21(14,0)$ & $59(39,3)$ \\
$\begin{array}{l}\text { 8. He cambiado mi prac- } \\
\text { tica cuando he encontra- } \\
\text { do evidencia al respecto }\end{array}$ & $70(46,7)$ & $80(53,3)$ & 0 \\
\hline
\end{tabular}

Tabla 5. Respuestas en el dominio habilidades y competencias de la versión Argentina del cuestionario EBPQ-19 de las licenciadas en obstetricia matriculadas en el Colegio de Obstétricas $V$ del partido de Quilmes, Buenos Aires ( $\mathrm{N}=150)$. Nota: valor mínimo=1, valor máximo $=7$.

\begin{tabular}{|c|c|c|c|c|}
\hline Pregunta & $\begin{array}{l}\text { Puntaje } \\
7, n \\
(\%) \\
\end{array}$ & $\begin{array}{l}\text { Puntaje } \\
6, n \\
(\%) \\
\end{array}$ & $\begin{array}{l}\text { Puntaje } \\
5, n \\
(\%) \\
\end{array}$ & $\begin{array}{c}\text { Puntaje } 4 \\
\text { o inferior, } \\
\mathrm{n}(\%) \\
\end{array}$ \\
\hline $\begin{array}{l}\text { 9. Habilidades con } \\
\text { las tecnologías de la } \\
\text { información }\end{array}$ & 0 & $\begin{array}{c}71 \\
(47,3)\end{array}$ & $7(4,7)$ & $72(48,0)$ \\
\hline $\begin{array}{l}\text { 10. Monitorización y } \\
\text { revisión de habilida- } \\
\text { des practicas }\end{array}$ & 0 & $\begin{array}{c}72 \\
(48,0)\end{array}$ & $\begin{array}{c}55 \\
(36,7)\end{array}$ & $23(15,3)$ \\
\hline $\begin{array}{l}\text { 11. Conversión de } \\
\text { mis necesidades } \\
\text { de información en } \\
\text { preguntas de investi- } \\
\text { gación }\end{array}$ & $\begin{array}{c}31 \\
(20,7)\end{array}$ & $\begin{array}{c}41 \\
(27,3)\end{array}$ & $\begin{array}{c}31 \\
(20,7)\end{array}$ & $47(31,3)$ \\
\hline $\begin{array}{l}\text { 12. Estar al dia con } \\
\text { los principales tipos } \\
\text { de información y sus } \\
\text { fuentes }\end{array}$ & $\begin{array}{c}31 \\
(20,7)\end{array}$ & $\begin{array}{c}40 \\
(26,7)\end{array}$ & $\begin{array}{c}57 \\
(38,0)\end{array}$ & $22(14,7)$ \\
\hline $\begin{array}{l}\text { 13. Capacidad para } \\
\text { analizar críticamente } \\
\text { la evidencia mediante } \\
\text { criterios explícitos }\end{array}$ & 0 & $2(1,3)$ & $\begin{array}{c}99 \\
(66,0)\end{array}$ & $49(32,7)$ \\
\hline $\begin{array}{l}\text { 14. Capacidad para } \\
\text { determinar la validez } \\
\text { del material encontra- } \\
\text { do (aplicabilidad clíni- } \\
\text { ca) }\end{array}$ & 0 & $\begin{array}{c}30 \\
(20,0)\end{array}$ & $\begin{array}{c}98 \\
(65,3)\end{array}$ & $22(14,7)$ \\
\hline $\begin{array}{l}\text { 15. Capacidad para } \\
\text { aplicar la información } \\
\text { encontrada a casos } \\
\text { concretos }\end{array}$ & $\begin{array}{c}30 \\
(20,0)\end{array}$ & $\begin{array}{c}30 \\
(20,0)\end{array}$ & $\begin{array}{c}68 \\
(45,3)\end{array}$ & $22(14,7)$ \\
\hline
\end{tabular}




\section{Dominio habilidades y competencias}

En la Tabla 5 se resumen las respuestas a los enunciados de los ítems vinculados al dominio 'habilidades y competencias'.

\section{Discusión}

A través de esta investigación pudimos obtener una versión adaptada al español que se habla en el área rioplatense de Argentina del cuestionario EBPQ-19 para evaluar la actitud de los profesionales de la salud hacia la PCBE. Además, pudimos documentar que, en la región evaluada por esta investigación, las licenciadas en obstetricia encuestadas muestran un gran compromiso para con las pacientes en cuanto a la consulta de información para resolver dudas durante la atención profesional, si bien tienen poca confianza en sus propias habilidades y competencias para ofrecer una PCBE. Nuestros resultados resultan concordantes con los obtenidos en otros estudios, como el de Díaz et al ${ }^{29}$ en el que las actitudes de los participantes hacia la PCBE mostraron la puntuación media más alta $(5,42)$, seguidas de los conocimientos/habilidades $(3,61)$ y la práctica $(3,40)$. Otros investigadores ${ }^{30}$ documentaron las barreras percibidas por personal de enfermería para aplicar una PCBE. Entre las principales se encontraban que las enfermeras consideraban que tenían poco tiempo para leer investigaciones ( $42 \%$ ), que no tenían la autoridad suficiente para cambiar procedimientos de atención al paciente (35\%), ni tiempo suficiente en el trabajo para poner en práctica nuevas ideas (36\%) y/o cambiar procedimientos de atención al paciente
( $35 \%)$. Vale destacar que otros estudios ${ }^{31}$, permitieron observar que el puntaje promedio más alto de las actitudes de los educadores en enfermería hacia la PCBE se da en las personas que tenían un doctorado en ciencias de enfermería.

Otras investigaciones había permitido generar diferentes espacios de diálogo donde los actores de las prácticas clínicas habían participado individualmente o en grupo de iguales, expresando libremente sus vivencias y experiencias ${ }^{32,33}$. Los diálogos y las narrativas han facilitado entrar de lleno en las diferentes vivencias de una misma realidad a través de la reflexión y el debate ${ }^{34,35}$. De este modo se ha obtenido una visión holística del aprendizaje clínico desde diferentes miradas y roles que ha facilitado interpretar el significado de la acción educativa.

Consideramos que la evaluación de las prácticas clínicas constituye un elemento importante del aprendizaje y facilita valorar la adquisición de las competencias ${ }^{36}$

Como limitaciones de esta investigación cabe señalar que los resultados observados al aplicar el cuestionario no son generalizables a otras profesionales, debido a que provienen de una muestra obtenida por conveniencia.

\section{Conclusiones}

La versión adaptada al español rioplatense de Argentina del cuestionario EBPQ-19 tiene un adecuado desempeño y alentamos su utilización para la evaluación y el monitoreo de programas educativos que promuevan la PCBE.

\section{Referencias}

1. Tyzio R, Cossart R, Khalilov I, Minlebaev M, Hübner CA, Represa A, et al. Maternal oxytocin triggers a transient inhibitory switch in GABA signaling in the fetal brain during delivery. Science. 2006;314(5806):1788-1792. Available from: 10.1126/science.1133212.

2. Phaneuf S, Linares BR, TambyRaja R, MacKenzie I, Bernal AL. Loss of myometrial oxytocin receptors during oxytocin-induced and oxytocinaugmented labour. Reproduction. 2000;120(1):91-97. Available from: 10.1530/iff.0.1200091.

3. Thomas TA, Fletcher JE, Hill RG. Influence of medication, pain and progress in labour on plasma beta-endorphin-like immunoreactivity. British journal of anaesthesia. 1982 apr;54(4):401-8. Available from: 10.1093/bja/54.4.401.

4. ACOG technical bulletin. Obstetric analgesia and anesthesia. Number 225-July 1996 (replaces No. 112, January 1988). Committee on Technical Bulletins of the American College of Obstetricians and Gynecologists. International journal of gynaecology and obstetrics: the official organ of the International Federation of Gynaecology and Obstetrics. 1996;54(3):281-292. Available from: http://www.ncbi.nlm.nih.gov/pubmed/8889638.

5. Browning AJ, Butt WR, Lynch SS, Shakespear RA, Crawford JS. Maternal and cord plasma concentrations of beta-lipotrophin, beta-endorphin and gamma-lipotrophin at delivery; effect of analgesia. Br J Obstet Gynaecol. 1983;90(12):1152-1156.

6. Lieberman E, O'Donoghue C. Unintended effects of epidural analgesia during labor: A systematic review. In: American Journal of Obstetrics and Gynecology. vol. 186; 2002.Available from: 10.1067/mob.2002.122522.

7. Krehbiel D, Poindron P, Lévy F, Prud'Homme MJ. Peridural anesthesia disturbs maternal behavior in primiparous and multiparous parturient ewes. Physiology and Behavior. 1987:40(4):463-472. Available from: 10.1016/0031-9384(87)90031-X.

8. Behrens O, Goeschen K, Luck HJ, Fuchs AR. Effects of lumbar epidural analgesia on prostaglandin $\mathrm{F} 2 \$ \alpha \$$ release and oxytocin secretion during labor. Prostaglandins. 1993;45(3):285-296. Available from: 10.1016/0090-6980(93)90053-A.

9. Neumark J, Hammerle AF, Biegelmayer C. Effects of epidural analgesia on plasma catecholamines and cortisol in parturition. Acta anaesthesiologica Scandinavica. 1985 aug;29(6):555-9. Available from: 10.1111/j.1399-6576.1985.tb02253.x.

10. Hale TW. Medications and Mother's Milk; 2008.

11. Jordan S, Emery S, Bradshaw C, Watkins A, Friswell W. The impact of intrapartum analgesia on infant feeding. BJOG: An International Journal of Obstetrics and Gynaecology. 2005;112(7):927-934. Available from: 10.1111/j.1471-0528.2005.00548.x.

12. Segal S, Csavoy AN, Datta S. The tocolytic effect of catecholamines in the gravid rat uterus. Anesthesia and Analgesia. 1998;87(4):864-869. Available from: 10.1097/00000539-199810000-00022.

13. Lederman RP, Lederman E, Work B, McCann DS. Anxiety and epinephrine in multiparous women in labor: relationship to duration of labor and fetal heart rate pattern. American journal of obstetrics and gynecology. 1985 dec;153(8):870-7. Available from: 10.1016/0002-9378(85)90692-1.

14. Jacobson B, Nyberg K, Grönbladh L, Eklund G, Bygdeman M, Rydberg U. Opiate addiction in adult offspring through possible imprinting after obstetric treatment. BMJ (Clinical research ed). 1990 nov;301(6760):1067-70. Available from: 10.1136/bmj.301.6760.1067.

15. Deneux-Tharaux C, Carmona E, Bouvier-Colle MH, Bréart G. Postpartum maternal mortality and cesarean delivery. Obstetrics and Gynecology. 2006;108(3):541-548. Available from: 10.1097/01.AOG.0000233154.62729.24

16. Levine EM, Ghai V, Barton JJ, Strom CM. Mode of delivery and risk of respiratory diseases in newborns. Obstetrics and gynecology. 2001 mar;97(3):439-42. Available from: 10.1016/s0029-7844(00)01150-9.

17. Brinsmead M, Smith R, Singh B, Lewin T, Owens P. Peripartum Concentrations of Beta Endorphin and Cortisol and Maternal Mood States. Australian and New Zealand Journal of Obstetrics and Gynaecology. 1985;25(3):194-197. Available from: 10.1111/j.1479-828X.1985.tb00642.x.

18. Hemminki E, Meriläinen J. Long-term effects of cesarean sections: ectopic pregnancies and placental problems. American journal of obstetrics and gynecology. 1996;174(5):1569-74. Available from: 10.1016/s0002-9378(96)70608-7.

19. Larguía AM, Lomuto CC, González MA. Guía para transformar maternidades tradicionales en maternidades centradas en la familia. 1st ed. Buenos Aires, Argentina: Fundación Neonatológica para el Recién Nacido y su Familia; 2006.

20. Resolución de Consejo Superior $n^{\circ}$ 1469/94. Complementación Curricular, Licenciatura en obstetricia.; 1994. Available from: https://www.fmed.uba. ar/index.php/carreras/licenciatura-en-obstetricia/complementacion-curricular.

21. McGe DL. Evidence-Based Medicine and Clinical Guidelines. In: MSD Manual Professional Version. Kenilworth, NJ, USA: Merck Sharp \& Dohme Corp; 2018.Available from: https://www.msdmanuals.com/professional/special-subjects/clinical-decision-making/evidence-based-medicine-andclinical-guidelines. 
22. Shaneyfelt T, Baum KD, Bell D, Feldstein D, Houston TK, Kaatz S, et al. Instruments for Evaluating Education. Jama. 2006;296(9). Available from: 10.1016/j.jirobp.2011.09.054.

23. Upton D, Upton P. Development of an evidence-based practice questionnaire for nurses. Journal of Advanced Nursing. 2006;53(4):454-458. Available from: 10.1111/j.1365-2648.2006.03739.x.

24. de Pedro Gómez J, Morales-Asencio JM, Abad AS, Veny MB, Roman MJR, Ronda FM. [Validation of the Spanish version of the Evidence Based Practice Questionnaire in Nurses]. Revista espanola de salud publica. 2009;83(4):577-86. Available from: 10.1590/s1135-57272009000400009.

25. Wild D, Grove A, Martin M, Eremenco S, McElroy S, Verjee-Lorenz A, et al. Principles of good practice for the translation and cultural adaptation process for patient-reported outcomes (PRO) measures: Report of the ISPOR Task Force for Translation and Cultural Adaptation. Value in Health. 2005;8(2):94-104. Available from: 10.1111/j.1524-4733.2005.04054.x.

26. Ramada-Rodilla JM, Serra-Pujadas C, Delclós-Clanchet GL. Adaptación cultural y validación de cuestionarios de salud: revisión y recomendaciones metodológicas. Salud Pública Méx. 2013;55(1):57-66. Available from: http://www.scielo.org.mx/scielo.php?script=sci_arttext\&pid=S003636342013000100009.

27. Streiner DL. Figuring out factors: the use and misuse of factor analysis. Canadian journal of psychiatry Revue canadienne de psychiatrie. 1994 apr;39(3):135-40. Available from: 10.1177/070674379403900303.

28. Hayes BE. Measuring Customer Satisfaction And Loyalty: Survey Design, Use, And Statistical Analysis Methods. 3rd ed. New Age International; 1998.

29. Martínez-Díaz JD, Jiménez-Rodríguez D, Muñoz-Ronda FJ, Aguilera-Manrique G, Ángeles López-Valverde M, del Mar Rodríguez-Salvador M. Competencia de las enfermeras de cuidados críticos para integrar y aplicar la práctica basada en la evidencia. Metas de Enfermería. 2014;17(3):20-26. Available from: https://www.enfermeria21.com/revistas/metas/articulo/80568/.

30. Straka KL, Brandt P, Brytus J. Brief report: Creating a culture of evidence-based practice and nursing research in a pediatric hospital. Journal of pediatric nursing;28(4):374-8. Available from: 10.1016/j.pedn.2012.08.001.

31. Hussein AHM, Hussein RG. The attitudes and barriers towards evidence-based practice among nursing educators. Journal of American Science. 2013;9(12):609-618. Available from: https://pdfs.semanticscholar.org/3bc0/98c8b9d852d460198bdb8ba96dc0c2504ca0.pdf.

32. Cossette S. De la recherche exploratoire à la recherche appliquée en sciences infirmières : complémentarités et finalités. Recherche en soins infirmiers. 2010;3(102):73-82. Available from: https:/www.cairn.info/resume.php?ID_ARTICLE=RSI_102_0073\&contenu=resume.

33. i Caparà NR, Morera-Pomarede MJ, Monteagudo CV, i Torras MH, Insa-Calderón E, Hurtado-Pardos $\mathrm{B}$, et al.. El Tutelatge de pràctiques, una eina per a la formació de l'estudiant. A 'Taula C: Tutorització dels estudiants'. Universitat de Girona. Institut de Ciències de l'Educació Josep Pallach; 2009. Available from: http://hdl.handle.net/10256/2002.

34. Àngels Domingo-Roget. El profesional reflexivo (D.A. Schön). Descripción de las tres fases del pensamiento práctico; 2019 . Available from: https: //practicareflexiva.pro/wp-content/uploads/2019/03/D.SCHON_FUNDAMENTOS.pdf.

35. Schön DA. La formación de profesionales reflexivos: Hacia un nuevo diseño de la enseñanza y el aprendizaje en las profesiones. Barcelona, España: Paidós; 1992.

36. Helminen K, Tossavainen K, Turunen H. Assessing clinical practice of student nurses: Views of teachers, mentors and students. Nurse Education Today. 2014;34(8):1161-1166. Available from: 10.1016/j.nedt.2014.04.007. 